\title{
Role of AMP-activated protein kinase in mechanism of metformin action
}

Rapid Publication

See related Commentary

on pages 1105-1107.

\author{
Gaochao Zhou, ${ }^{1}$ Robert Myers, ${ }^{1}$ Ying Li, ${ }^{1}$ Yuli Chen, ${ }^{1}$ \\ Xiaolan Shen, ${ }^{1}$ Judy Fenyk-Melody, ${ }^{1}$ Margaret Wu, ${ }^{1}$ John Ventre, ${ }^{1}$ \\ Thomas Doebber, ${ }^{1}$ Nobuharu Fujii, ${ }^{2}$ Nicolas Musi, ${ }^{2}$ \\ Michael F. Hirshman, ${ }^{2}$ Laurie J. Goodyear, ${ }^{2}$ and David E. Moller ${ }^{1}$ \\ ${ }^{1}$ Departments of Molecular Endocrinology, Metabolic Disorders, and Comparative Medicine, \\ Merck Research Laboratories, Rahway, New Jersey, USA \\ ${ }_{2}^{2}$ Joslin Diabetes Center and Harvard Medical School, Boston, Massachusetts, USA
}

Address correspondence to: Gaochao Zhou, Merck Research Laboratories, Rahway, New Jersey 07065, USA.

Phone: (732) 594-4782; Fax: (732) 594-5700; E-mail: gaochao_zhou@merck.com.

Received for publication June 13, 2001, and accepted in revised form August 28, 2001.

Metformin is a widely used drug for treatment of type 2 diabetes with no defined cellular mechanism of action. Its glucose-lowering effect results from decreased hepatic glucose production and increased glucose utilization. Metformin's beneficial effects on circulating lipids have been linked to reduced fatty liver. AMP-activated protein kinase (AMPK) is a major cellular regulator of lipid and glucose metabolism. Here we report that metformin activates AMPK in hepatocytes; as a result, acetyl-CoA carboxylase (ACC) activity is reduced, fatty acid oxidation is induced, and expression of lipogenic enzymes is suppressed. Activation of AMPK by metformin or an adenosine analogue suppresses expression of SREBP-1, a key lipogenic transcription factor. In metformin-treated rats, hepatic expression of SREBP-1 (and other lipogenic) mRNAs and protein is reduced; activity of the AMPK target, ACC, is also reduced. Using a novel AMPK inhibitor, we find that AMPK activation is required for metformin's inhibitory effect on glucose production by hepatocytes. In isolated rat skeletal muscles, metformin stimulates glucose uptake coincident with AMPK activation. Activation of AMPK provides a unified explanation for the pleiotropic beneficial effects of this drug; these results also suggest that alternative means of modulating AMPK should be useful for the treatment of metabolic disorders.

This article was published online in advance of the print edition.

The date of publication is available from the JCI website, http://www.jci.org.

J. Clin. Invest. 108:1167-1174 (2001). DOI:10.1172/JCI200113505.

\section{Introduction}

Metformin is widely used for the therapy of type 2 diabetes mellitus (DM2) (1). Metformin ameliorates hyperglycemia without stimulating insulin secretion, promoting weight gain, or causing hypoglycemia $(2,3)$. In addition, metformin has beneficial effects on circulating lipids linked to increased cardiovascular risk (2-4).

Although used as a drug since 1957, the mechanism(s) by which metformin lowers glucose and lipids remains an enigma. Two effects, decreased hepatic glucose production $(2,5,6)$ and increased skeletal myocyte glucose uptake $(7,8)$, have been implicated as major contributors to glucose-lowering biosynthetic pathways due to its role in the phosphorylation and inactivation of key enzymes such as acetyl-CoA carboxylase (ACC) (10). More recent data strongly suggest that AMPK has a wider role in metabolic regulation $(10$, 11): this includes fatty acid oxidation, muscle glucose uptake (12-14), expression of cAMP-stimulated gluconeogenic genes such as PEPCK and G6Pase (15), and glucose-stimulated genes associated with hepatic lipogenesis, including fatty acid synthase (FAS), Spot-14 (S14), and L-type pyruvate kinase (16). Chronic activation of AMPK may also induce the expression of muscle hexokinase and glucose transporters (Glut4), mimicking the effects of extensive exercise training (17). Thus, it has been predicted that AMPK activation would be a good approach to treat DM2 (11). In this report we tested the hypothesis that activation of AMPK mediates the beneficial metabolic effects of metformin.

efficacy. Metformin also decreases hepatic lipids in obese mice (9). Metformin is, however, a low-potency compound that is used at high doses, resulting in only modest net efficacy; in addition, significant side effects can occur (2). Thus, an understanding of the molecular basis for metformin's effects on glucose and lipid homeostasis is a critical focus of research directed toward improved therapeutic approaches to DM2 and related disorders.

AMP-activated protein kinase (AMPK) provides a candidate target capable of mediating the beneficial metabolic effects of metformin. AMPK is a multisubunit enzyme that is recognized as a major regulator of lipid

\section{Methods}

Measurements of AMPK, ACC, and fatty acid oxidation in primary hepatocytes. Hepatocytes were isolated from male Sprague Dawley (SD) rats by collagenase digestion (18). For the AMPK assay, cells were seeded in six-well plates at $1.5 \times 10^{6}$ cells/well in DMEM containing $100 \mathrm{U} / \mathrm{ml}$ penicillin, $100 \mu \mathrm{g} / \mathrm{ml}$ streptomycin, $10 \% \mathrm{FBS}, 100 \mathrm{nM}$ insulin, $100 \mathrm{nM}$ dexamethasone, and $5 \mu \mathrm{g} / \mathrm{ml}$ cultured in serum-free DMEM for 16 hours followed by treatment for 1 hour or 7 hours with control medium, 5-amino-imidazole carboxamide ribotransferrin for 4 hours. Cells were then 
side (AICAR), or metformin at concentrations indicated. For a 39-hour treatment, cells for both control and metformin $(10$ or $20 \mu \mathrm{M})$ groups were cultured in DMEM plus 5\% FBS and $100 \mathrm{nM}$ insulin, and the fresh control and metformin-containing medium were replaced every 12 hours (last medium change was 3 hours before harvest). After treatment, the cells were directly lysed in digitonin-containing and phosphatase inhibitor-containing buffer A (19), followed by precipitation with ammonium sulfate at $35 \%$ saturation. AMPK activity was determined by measurement of phosphorylation of a synthetic peptide substrate, SAMS (HMR-
SAMSGLHLVKRR) (20). For ACC assay, the $35 \%$ ammonium sulfate precipitate from digitonin-lysed hepatocytes $(4 \mu \mathrm{g}$ each) was used for determination of ACC activity via ${ }^{14} \mathrm{CO}_{2}$ fixation in the presence of $20 \mathrm{mM}$ citrate as done previously (19). For fatty acid oxidation, the oxidation of ${ }^{14} \mathrm{C}$-oleate to acid-soluble products was performed as done previously (21), but in medium M199 in the absence of albumin.

AMPK partial purification and in vitro kinase assay. Liver AMPK was partially purified from male SD rats (22) to the blue-Sepharose step. The $100-\mu$ reaction mixture contained $100 \mu \mathrm{M}$ AMP, $100 \mu \mathrm{M}$ ATP $\left(0.5 \mu \mathrm{Ci}^{33} \mathrm{P}-\mathrm{ATP}\right.$ per reac- tion), and $50 \mu \mathrm{M}$ SAMS in a buffer (40 mM HEPES, pH 7.0, 80 mM NaCl, 0.8 $\mathrm{mM}$ EDTA, $5 \mathrm{mM} \mathrm{MgCl}_{2}, 0.025 \%$ BSA, and $0.8 \mathrm{mM}$ DTT). The reaction was initiated with addition of the enzyme. After 30 -minute incubation at $30^{\circ} \mathrm{C}$, the reaction was stopped by addition of $80 \mu \mathrm{l} 1 \% \mathrm{H}_{3} \mathrm{PO}_{4}$. Aliquots $(100 \mu \mathrm{l})$ were transferred to 96-well MultiScreen plates (MAPHNOB50; Millipore Corp., Bedford, Massachusetts, USA). The plate was washed three times with $1 \%$ $\mathrm{H}_{3} \mathrm{PO}_{4}$ followed by detection in a Topcount. The in vitro AMPK inhibition data obtained with compound C - (6[4-(2-Piperidin-1-yl-ethoxy)-phenyl)]-3pyridin-4-yl-pyyrazolo[1,5-a] pyrimi- $\mathbf{a}$

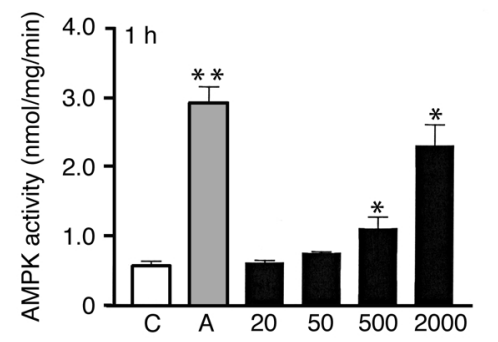

Metformin $(\mu \mathrm{M})$

\section{b}

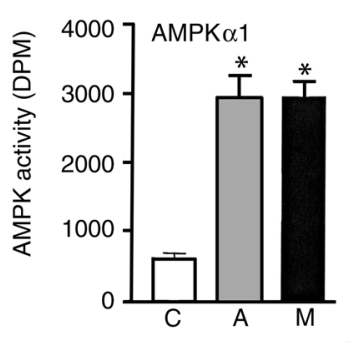

d

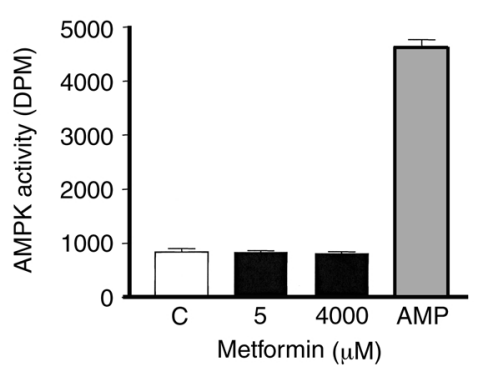

$\mathbf{f}$

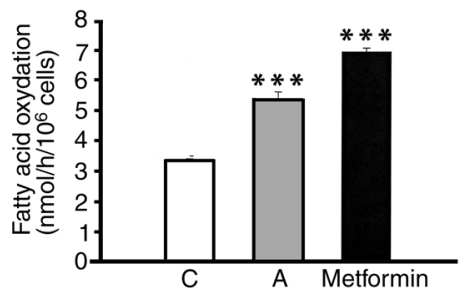

e
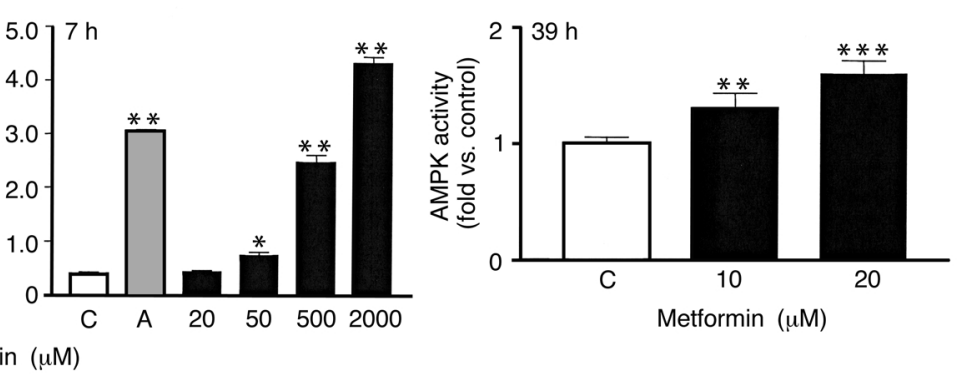

C
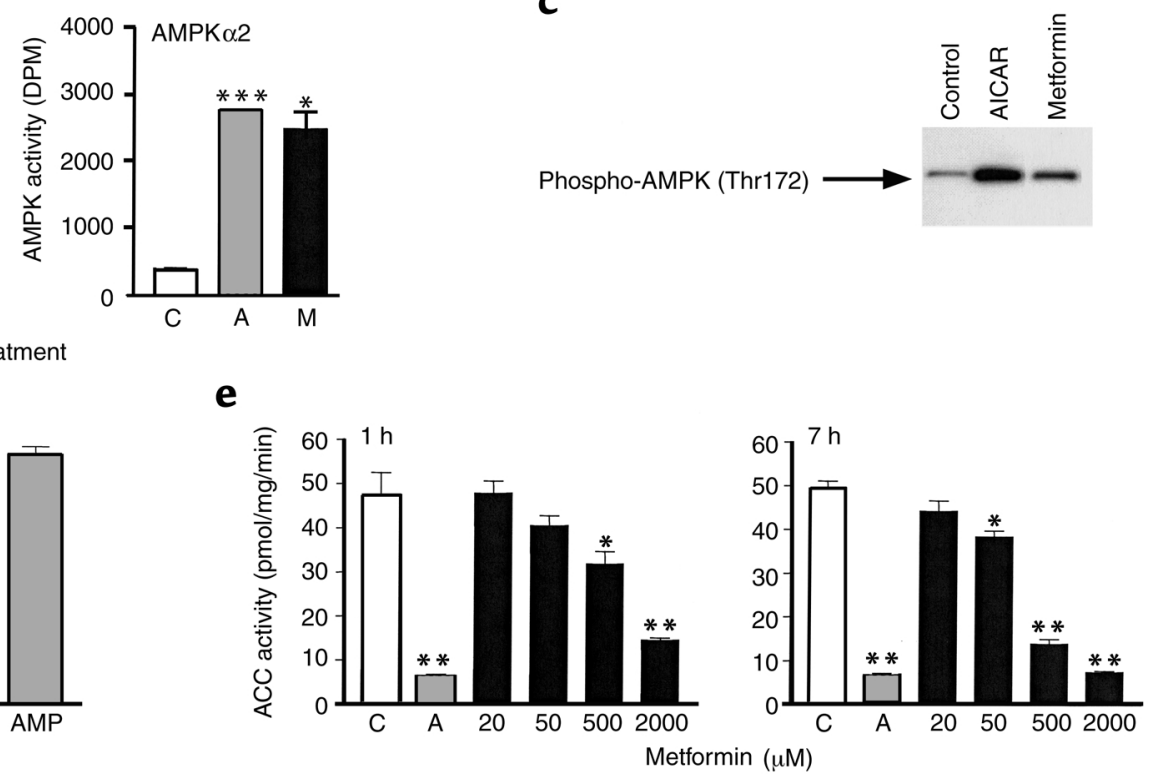

Figure 1

Metformin mediates AMPK activation in primary hepatocytes. (a) Metformin (black bars) and $\operatorname{AICAR}(\mathrm{A} ; 500 \mu \mathrm{M})$ activate AMPK in rat primary hepatocytes. The treatments were 1 hour, 7 hours, and 39 hours, respectively. (b) Metformin $(500 \mu \mathrm{M})$ and $\operatorname{AICAR}(500 \mu \mathrm{M})$ activated both AMPK $\alpha 1$ and AMPK $\alpha 2$ complexes demonstrated by immunoprecipitation-AMPK assay. DPM, disintegrations per minute. (c) Metformin $(1 \mathrm{mM})$ and AICAR $(500 \mu \mathrm{M})$ stimulated AMPK Thr172 phosphorylation. (d) Metformin does not activate partially purified rat liver AMPK in vitro. (e) Metformin and AICAR $(500 \mu \mathrm{M})$ inactivate ACC in rat primary hepatocytes. (f) Metformin $(500 \mu \mathrm{M}, 4$ hours $)$ and $\operatorname{AICAR}(500 \mu \mathrm{M}, 4$ hours $)$ stimulate hepatocyte fatty acid oxidation. $C$, vehicle control. Mean ( $n=3$ wells per treatment for 1 hour and 7 hours; for 39 -hour treatment, $n=12-15$ wells per treatment) \pm SEM values are shown. ${ }^{*} P<0.05,{ }^{*} P<0.01$, *** $P<0.001$ vs. control medium (paired $t$ test). 

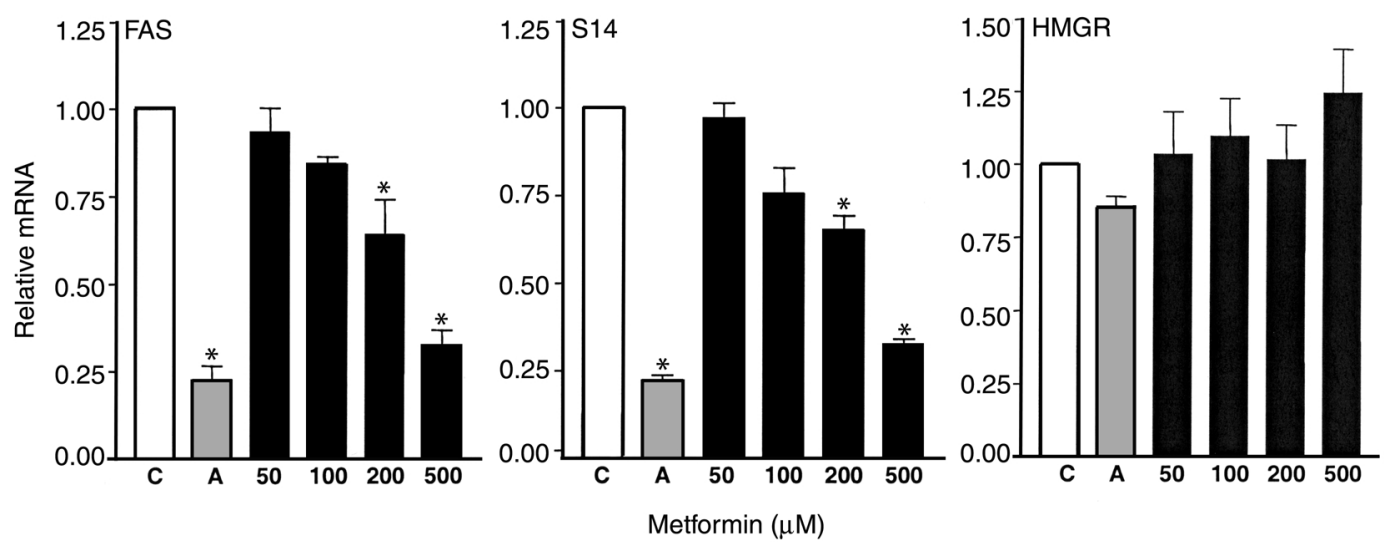

\section{Figure 2}

Treatment of hepatocytes with metformin or AICAR suppresses lipogenic genes in rat primary hepatocytes. Taqman-based real-time RT-PCR was used to quantitate mRNAs for FAS, S14, and HMGR. Mean ( $n=3$ per treatment) \pm SEM values are shown. ${ }^{*} P<0.05$, ${ }^{*} P<0.01$ vs. control medium (paired $t$ test). C, control; A, positive control (AICAR, $\left.500 \mu \mathrm{M}\right)$. These experiments were repeated a minimum of three times with similar results.

dine - were fit to the following equation for competitive inhibition by nonlinear regression using a least-squares Marquardt algorithm in a computer program written by N. Thornberry of Merck Research Laboratories: $V_{\mathrm{i}} / V_{\mathrm{o}}=$ $\left(K_{\mathrm{m}}+S\right) /\left[S+K_{\mathrm{m}} \times\left(1+I / K_{\mathrm{i}}\right)\right]$, where $V_{\mathrm{i}}$ is the inhibited velocity, $V_{\mathrm{o}}$ is the initial velocity, $S$ is the substrate (ATP) concentration, $K_{\mathrm{m}}$ is the Michaelis constant for ATP, $I$ is the inhibitor (compound C) concentration, and $K_{\mathrm{i}}$ is the dissociation constant for compound C.

Quantitation of $m R N A$. Rat hepatocytes were seeded and starved in M199 medium as described (16). Cells were then treated for 6 hours with the same serum-free and hormone-containing medium containing $25 \mathrm{mM}$ glucose in the presence or absence of AICAR or increasing concentrations of metformin as indicated. Total RNAs were extracted from cultured hepatocytes using the guanidine thiocyanate method (TRIZOL; Life Technologies Inc., Gaithersburg, Maryland, USA). The mRNA was quantitated using a TaqMan One Step Gold RT-PCR kit (Applied Biosystems, Branchburg, New Jersey, USA). Primers were as follows: for S14, S14p (6FAM-TGGTGATGATCCCCAGCCTTCTGAG), S14F (TGTGGTGCGGAACATGGA), and S14R (CTCCGGACCCACTCAGCTC); for FAS, FASp (6FAM-TCCGCC AGAGCCCTTTGTTAATTGG), FASF (AACTGAACGGCATTACTCGGTC), and FASR (GTGTCCCATGTTGGATTTGGT); for sterol regulatory element-binding protein 1 (SREBP1),
SREBP1P (6FAM-TCCACCATCGGCACCCACTGCT), SREBP1F (AGGACCCAAGGTGACACCTG), SREBP1R (GCCGGACGGG-TACATCTTT).

Hepatocyte glucose production. Hepatocytes from 24-hour starved rats were incubated at $2 \times 10^{6} \mathrm{cell} / \mathrm{ml}$ in bicarbonate-buffered saline medium containing $10 \mathrm{mM}$ L-lactate, $1 \mathrm{mM}$ pyruvate, $0.3 \mu \mathrm{M}$ glucagon, with a gas phase of $\mathrm{O}_{2} / \mathrm{CO}_{2}$ (19:1). Glucose was measured using glucose oxidase kit from Sigma Chemical Co. (St. Louis, Missouri, USA).

Measurements of muscle AMPK activity and glucose uptake. Isolated rat epitrochlearis muscles were incubated for 3 hours with metformin $(2 \mathrm{mM})$ or control medium followed by measurement of AMPK $\alpha 1$ or AMPK $\alpha 2$ activities as described (23). For glucose uptake, insulin $(300 \mathrm{nM})$ was present where indicated for the last 30 minutes of the 3-hour incubation. Then, 3-0-methylglucose uptake was measured using a 10-minute incubation in the absence or presence of metformin and/or insulin as described previously (23).

Animal experiments. Oral gavage was used to administer $1 \mathrm{ml}$ of metformin $(100 \mathrm{mg} / \mathrm{ml})$ or water alone to male SD rats (300-350 g, $n=7-8)$. Rats were treated once (see Table 1and Figure $5 \mathrm{~b}$ ) or twice (see Figure 6) a day for 5 days. Rats were starved for 20 hours and then re-fed for 2 hours before the final dose; 4 hours after final dose, the animals were anesthetized and livers rapidly removed by freeze clamping fol- lowed by blood withdrawal. RNA was prepared from the freeze-clamped liver by Ultraspec RNA isolation reagent (Biotecx Laboratories Inc., Houston, Texas, USA). Nuclear extracts were prepared from a pool of seven rat livers (24). Glucose levels were determined using the standard glucose oxidase assay kit; $\beta$-hydroxybutyrate concentrations were assayed by measuring the reduction of NAD to NADH with a standard assay kit (Sigma Chemical Co.). FFA levels were measured with the assay kit from Boehringer Mannheim GmbH (Mannheim, Germany). Triglyceride levels were assayed with a kit from Roche Diagnostics (Indianapolis, Indiana, USA). Insulin concentrations were measured with the enzyme-immunoassay kit from ALPCO Ltd. (Windham, New Hampshire, USA). All in vivo experiments were approved by the Institutional Animal Care and Use Committee.

Immunoblot analysis. For phosphoAMPK (Thr172) detection, the 35\% ammonium sulfate precipitate from treated rat hepatocytes was used for Western blot analysis using polyclonal Ab's to phospho AMPK $\alpha$ (Thr172; Cell Signaling Technology, Beverly, Massachusetts, USA). For SREBP-1, $100 \mu \mathrm{g}$ nuclear extracts from metformin-treated rat livers were similarly analyzed, but using monoclonal anti-SREBP-1 Ab (prepared from hybridoma CRL-2121; American Type Culture Collection [ATCC], Rockville, Maryland, USA). 


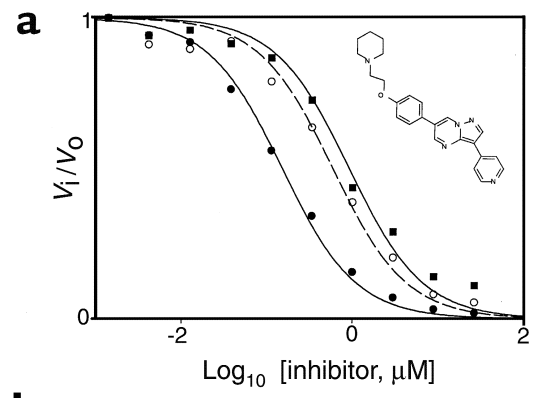

b

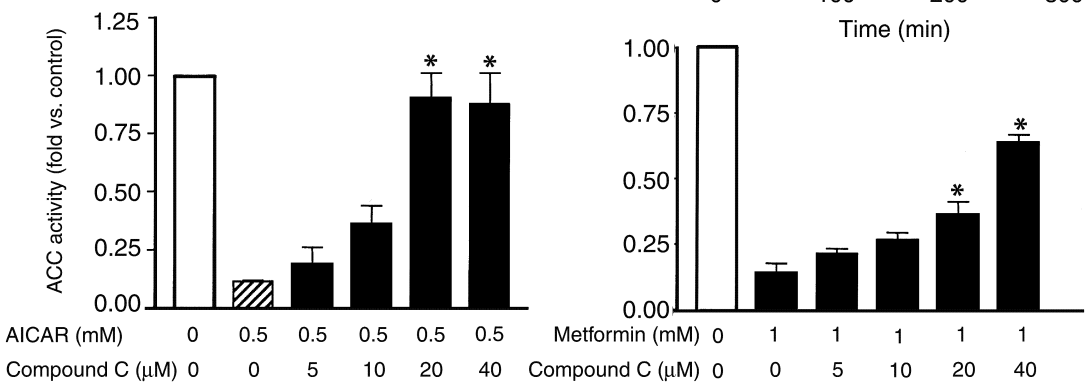

Figure 3

Discovery and use of a novel small-molecule AMPK inhibitor to establish that the effects of metformin on ACC and glucose production are AMPK dependent. (a) Inhibition of partially purified AMPK by compound C (inset shows the chemical structure) is reversible and competitive with respect to ATP. The in vitro kinase assay was performed in the presence of $5 \mu \mathrm{M}$ ATP, $0 \mu \mathrm{M}$ AMP (filled circles), $100 \mu \mathrm{M}$ ATP, $0 \mu \mathrm{M}$ AMP (filled squares), and $100 \mu \mathrm{M}$ ATP plus $100 \mu \mathrm{M}$ AMP (open circles). (b) Compound C inhibits the effects of AICAR and metformin on ACC in rat hepatocytes. Mean ( $n=3$ per treatment) \pm SEM values are shown. ${ }^{*} P<0.05$ vs. control medium (paired $t$ test). (c) Compound $C$ attenuates the ability of metformin to suppress glucagon-stimulated glucose production by hepatocytes from 24-hour starved rats. At time 0 , compound $\mathrm{C}(40 \mu \mathrm{M})$ was added, and at time 30 minutes, metformin ( $2 \mathrm{mM}$ ) was added. Squares, control; diamonds, metformin; circles, metformin plus compound $C$. Each point represents the mean \pm SEM of four replicate assays. ${ }^{*} P<0.05$, ${ }^{*} P<0.01 \mathrm{vs}$. metformin alone at the same time point (paired $t$ test). The experiment was repeated three times with similar results.

Immunoprecipitation-AMPK assay. Ten micrograms of $35 \%$ ammonium sulfate precipitate (containing AMPK) from AICAR- or metformin-treated rat hepatocytes was immunoprecipitated using polyclonal Ab's raised against AMPK $\alpha 1\left(\mathrm{NH}_{2}\right.$-DFYLATSPPDSFLDDHHLTR-OH) or AMPK $\alpha 2\left(\mathrm{NH}_{2}-\right.$ MDDSAMHIPPGLKPH-OH), followed by AMPK assay.

\section{Results}

Metformin promotes AMPK activation in primary rat hepatocytes. Although steadystate plasma levels of metformin in humans are reported to be around 10 $\mu \mathrm{M}(25,26)$ to as high as $40 \mu \mathrm{M}(27)$, studies in rats showed that levels in liver are severalfold higher than in plasma (28). Thus, liver levels of greater than $180 \mu \mathrm{M}$ can be achieved in rats after a $50 \mathrm{mg} / \mathrm{kg}$ dose (29); this dose is lower than doses typically required for efficacy in diabetic rats $(30,31)$. In addition to the fact that high concentrations may be achieved in target tissues, membrane permeability of metformin is a time-dependent and slow process (32). Therefore, we anticipated that high concentrations and/or longer-term experiments might be required in order to see effects of metformin on AMPK in cells or tissues. In primary cultured hepatocytes from both rats and humans, metformin activated AMPK in a concentration- and time-dependent manner (Figure 1a and data not shown). AICAR was used as positive control. AICAR is a cell-permeable adenosine analogue that can be phosphorylated to ZMP, an AMP analogue and known AMPK activator (10). After a 1-hour treatment, $500 \mu \mathrm{M}$ metformin was required to significantly activate AMPK, whereas after a 7-hour treatment, $50 \mu \mathrm{M}$ was sufficient to sig- nificantly activate the enzyme. Maximal AMPK stimulation, comparable to the effect of $500 \mu \mathrm{M}$ AICAR, was achieved with metformin concentrations of $2,000 \mu \mathrm{M}$ (1 hour) or $500 \mu \mathrm{M}$ ( 7 hours). To ensure that lower concentrations could mediate a similar effect, rat hepatocytes were incubated with $10 \mu \mathrm{M}$ or $20 \mu \mathrm{M}$ metformin for 39 hours. Both $10 \mu \mathrm{M}$ and $20 \mu \mathrm{M}$ metformin produced significant AMPK activation (1.3-fold, $P=0.0062$, and 1.6fold, $P=0.0007$, respectively; Figure 1a). Given the time-dependent effects of metformin, we relied on relatively high concentrations in several shorterterm experiments described below in order to augment the ability to see relevant effects. In Figure 1b, we used an immunoprecipitation-AMPK assay to show that metformin as well as AICAR activated AMPK in both AMPK $\alpha 1$ and AMPK $\alpha 2$ complexes efficiently.

AMP activates AMPK by promoting its phosphorylation at Thr 172 and by direct activation via an allosteric AMP site. Since enzyme activity shown in Figure 1, a and b, was measured after precipitation from hepatocyte lysates and in the presence of AMP, it is likely that metformin promotes AMPK phosphorylation. In keeping with this notion, we observed that treatment of hepatocytes with either metformin or AICAR resulted in slower electrophoretic mobility of AMPK $\alpha 1$ and AMPK $\alpha 2$, which is consistent with increased Ser/Thr phosphorylation (data not shown). More specifically, both metformin and AICAR treatment induced AMPK Thr172 phosphorylation demonstrated by using anti-phospho-AMPK Ab (Figure 1c).

Recent observations suggest that metformin can impair oxidative phosphorylation by inhibiting mitochondrial phosphorylation complex 1 (32, 33). Although some have reported that very high metformin concentrations $(10 \mathrm{mM})$ can suppress total cellular ATP levels $(25,33)$, Owen et al. (32) pointed out that more subtle changes in the free ATP/ADP ratio might occur with concentrations of metformin that do not suppress total ATP, but do inhibit gluconeogenesis. We also found that measured total ATP concentrations from metformin-treated 
(500-2,000 $\mu \mathrm{M})$ rat hepatocytes were not affected (data not shown). Thus, it is still plausible that metformin could produce a subtle decline in the free ATP/ADP and ATP/AMP ratios or a change in local concentrations of nucleotides, serving as a stimulus for AMPK phosphorylation and activation. Alternatively, metformin could directly activate AMPK kinase (AMPKK) or promote AMPK phosphorylation by binding to AMPK and making it a better substrate for AMPKK or worse substrate for phosphatase PP2C (10). The possibility that metformin could be a direct allosteric AMPK activator was excluded by showing that, when incubated with partially purified rat liver AMPK, metformin (from $4 \mu \mathrm{M}$ to $12 \mathrm{mM}$ ), unlike AMP, did not activate the enzyme (Figure 1d).

Metformin induces ACC inactivation in primary bepatocytes. As a result of AMPK activation, hepatocyte ACC activity was markedly suppressed by metformin treatment, paralleling concentrations and time points associated with AMPK activation (Figure 1e). ACC catalyzes the biosynthesis of malonyl-CoA from acetyl-CoA. Malonyl-CoA is an initial substrate for de novo fatty acid biosynthesis, and it serves as a potent inhibitor of carnitine palmitoyl transferase I (CPT-I), a rate-limiting step for fatty acid oxidation (34). Thus, decreased malonylCoA concentrations, as a result of ACC inactivation, could lead to decreased lipid synthesis and an increased rate of fatty acid oxidation. Indeed, like AICAR, metformin stimulated fatty acid oxidation in isolated rat hepatocytes (Figure 1f).

Metformin and AICAR suppress lipogenic gene expression. AMPK is also involved in gene regulation (10). As noted above, activation of AMPK can suppress the expression of several glucoseactivated lipogenesis-associated genes. Similar to the effect of AICAR, progressive inhibition of glucose-induced FAS and S14 expression was measured in hepatocytes in response to increasing concentrations of metformin (Figure 2). As a control, expression of HMG-CoA reductase (HMGR), a gene not affected by AICAR, was not affected by metformin.

Identification and characterization of AMPK inbibitor. To firmly establish the role of AMPK in mediating metformin's effects, a high-throughput in vitro assay was used to identify small-molecule AMPK inhibitors. Screening of a compound library containing more than 10,000 molecules lead to the discovery and characterization of compound C (inset in Figure 3a). Experiments conducted using variable ATP concentrations revealed that compound $\mathrm{C}$ is a potent reversible inhibitor that is competitive with ATP, with $K_{\mathrm{i}}=109 \pm 16 \mathrm{nM}$ in the absence of AMP (Figure 3a). In in vitro assays, compound $\mathrm{C}$ did not exhibit significant inhibition of several structurally related kinases including ZAPK, SYK, PKC $\theta$, PKA, and JAK3. Thus, to our knowledge, this is the first description of a potent and selective small-molecule AMPK inhibitor that may be useful as a means to further assess the physiologic effects of this pathway. Incubation of cultured hepatocytes with compound $\mathrm{C}$ inhibited ACC inactivation by either AICAR or metformin (Figure $3 \mathrm{~b}$ ). Compound $\mathrm{C}$ also attenuated AICAR and metformin's effect to increase fatty acid oxidation or suppress lipogenic genes in hepatocytes (not shown).

Metformin-mediated hepatocyte glucose production requires AMPK activation. It is believed that metformin-mediated inhibition of hepatic glucose production (HGP) plays a major role in its glucose-lowering efficacy $(2,3,6)$. Here, we determined that both metformin and AICAR were able to inhibit cumulative glucose production in primary cultured rat hepatocytes stimulated with glucagon (Figure $3 c$ and data not shown). Importantly, coincubation of hepatocytes with compound $\mathrm{C}$ was able to attenuate the effects of metformin to decrease glucose production in these cells (Figure $3 c$ ), demonstrating that AMPK activation was required for metformin's inhibitory effect on hepatocyte glucose production.

Metformin activates muscle AMPK and promotes glucose uptake. Since AMPK activation is implicated as a mechanism for stimulation of glucose
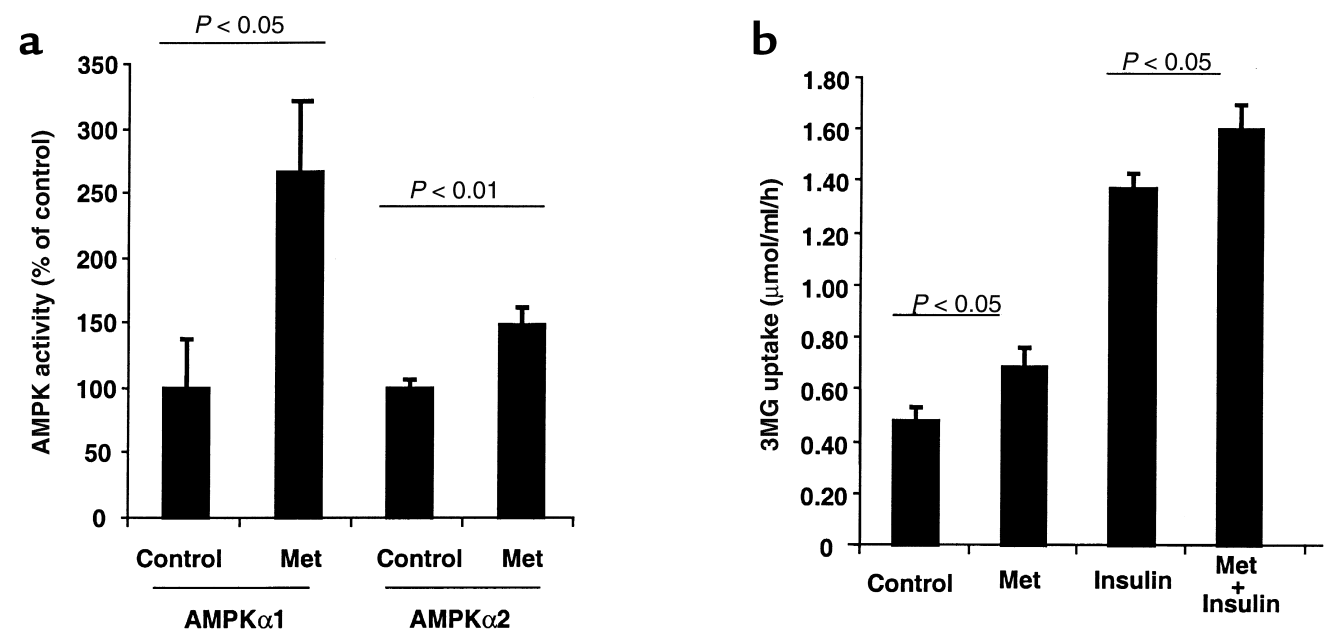

Figure 4

Metformin (Met; 2 mM, 3 hours) stimulates AMPK activity in skeletal muscle in association with induction of glucose uptake. (a) AMPK activity; (b) glucose uptake. Mean ( $n=5-6$ isolated rat epitrochlearis muscles per treatment $) \pm$ SEM values is shown. 


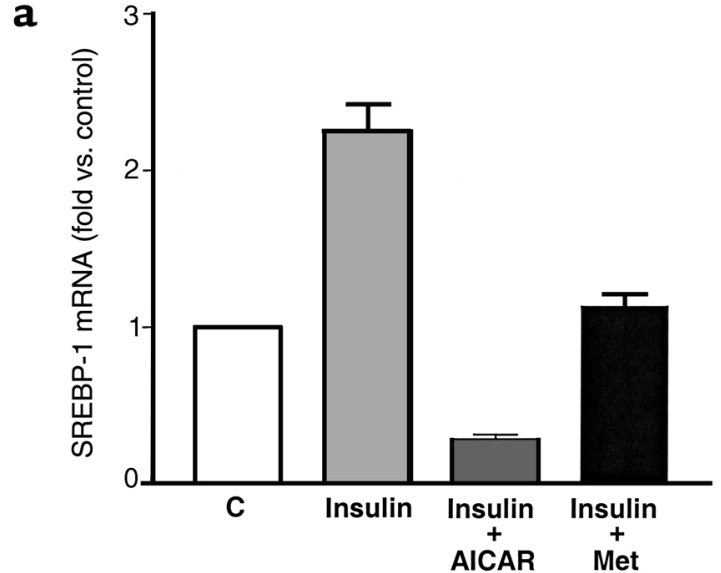

b

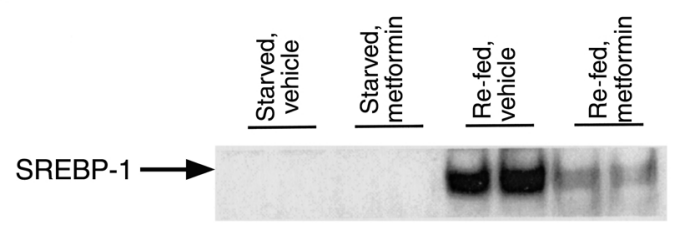

\section{Figure 5}

Metformin and AICAR downregulate hepatic SREBP-1. (a) Metformin $(500 \mu \mathrm{M})$ and AICAR $(500 \mu \mathrm{M})$ have similar effects to suppress SREBP-1 mRNA expression in rat hepatocytes. Mean \pm SEM ( $n=3$ replicate assays) are shown. Insulin significantly increased SREBP-1 mRNA ( $P=0.03$ vs. control medium by Student's $t$ test). Both AICAR and metformin significantly decreased SREBP-1 mRNA with $P$ values of 0.0046 and 0.01 , respectively, versus insulin. (b) Metformin prevents re-fed stimulated accumulation of mature SREBP-1 in nuclear extracts from treated rats. Western blot analysis using a monoclonal anti-SREBP-1 Ab (prepared from hybridoma CRL-2121) was performed. Similar results were obtained from a separate in vivo experiment.

uptake in skeletal muscle (12-14), we assessed the effect of metformin on glucose uptake and AMPK activity in intact rat epitrochlearis muscles. Incubation of isolated muscles with metformin resulted in an increase in the activity of both catalytic subunits of AMPK (Figure 4a). This was coincident with a significant increase in glucose uptake that was also observed to be additive with the effect of insulin stimulation (Figure 4b). In previous studies, AMPK $\alpha 2$ was stimulated to a greater extent than AMPK $\alpha 1$ by AICAR and other stress (23). Further studies will be required to assess this difference.

AMPK activation suppresses SREBP-1. SREBP-1c is an important insulinstimulated transcription factor that is implicated in the pathogenesis of insulin resistance, dyslipidemia, and DM2 $(35,36)$. Target genes that are induced by SREBP-1 include those that encode lipogenic enzymes, such as FAS and S14. Under conditions where SREBP-1 was induced by

\section{Table 1} activation responding values from vehicle-treated rats.
Assessment of metformin's in vivo effects. To assess whether selected effects of metformin described above also occurred in vivo, SD rats were studied (Table 1). Rats were orally dosed with metformin or vehicle $\left(\mathrm{H}_{2} \mathrm{O}\right)$ for 5 days. Rats were starved for 20 hours and then re-fed for 2 hours before the final dose. Four hours after the final dose, tissue and blood samples were obtained for analysis (see Methods). During starvation, there should be very little lipid synthesis. Upon refeeding, hepatic lipid synthesis should be dramatically induced. Metformin's effects were examined under re-fed conditions. Along with modest decreases in plasma insulin and triglycerides, a small, but significant increase in $\beta$-hydroxybutyrate was present, suggesting that hepatic fatty acid oxidation was induced in metformin-treated rats. Furthermore, metformin treatment produced significant decreases in hepatic expression of mRNAs for SREBP-1, FAS, and $\mathrm{S} 14$ that were consistent with effects documented in cells (Table 1 ). The mature SREBP-1 protein in rat liver nuclear extracts was examined using an anti-SREBP1 Ab (Figure 5b). As anticipated (35), SREBP-1 matureform protein was not detected in hepatic nuclear extracts from starved animals. In re-fed animals, mature SREBP-1 protein had accumulated consistent with an increase in lipid synthesis under this condition. Treat-

In vivo effects of metformin on selected parameters known to be downstream of AMPK

\begin{tabular}{|c|c|c|c|}
\hline \multirow[t]{6}{*}{ Serum } & & Vehicle & Metformin \\
\hline & Glucose (mg/dl) & $134.24 \pm 4.82$ & $126.33 \pm 7.19$ \\
\hline & $\mathrm{TG}(\mathrm{mg} / \mathrm{dl})$ & $40.97 \pm 2.81$ & $30.2 \pm 3.49^{\mathrm{A}}$ \\
\hline & FFA (mM) & $0.17 \pm 0.04$ & $0.2 \pm 0.04$ \\
\hline & Insulin (ng/ml) & $1.48 \pm 0.19$ & $0.49 \pm 0.09^{A}$ \\
\hline & $\beta$-hydroxybutyrate $(\mathrm{mg} / \mathrm{dl})$ & $0.94 \pm 0.06$ & $1.5 \pm 0.15^{\mathrm{A}}$ \\
\hline \multirow[t]{4}{*}{ RNA } & & Fold & Fold \\
\hline & SREBP-1 & $1 \pm 0.06$ & $0.5 \pm 0.03^{\mathrm{A}}$ \\
\hline & FAS & $1 \pm 0.06$ & $0.35 \pm 0.02^{\mathrm{A}}$ \\
\hline & S14 & $1 \pm 0.06$ & $0.43 \pm 0.03^{\mathrm{A}}$ \\
\hline
\end{tabular}

Normal SD rats were treated as described in Methods. In rats studied in the fed state, serum samples were analyzed for glucose, triglyceride (TG), FFA, insulin, and $\beta$-hydroxybutyrate. Hepatic mRNA levels were quantitated. Results are expressed as mean values from seven rats in each group. ${ }^{A} P<0.05$ vs. cor- 


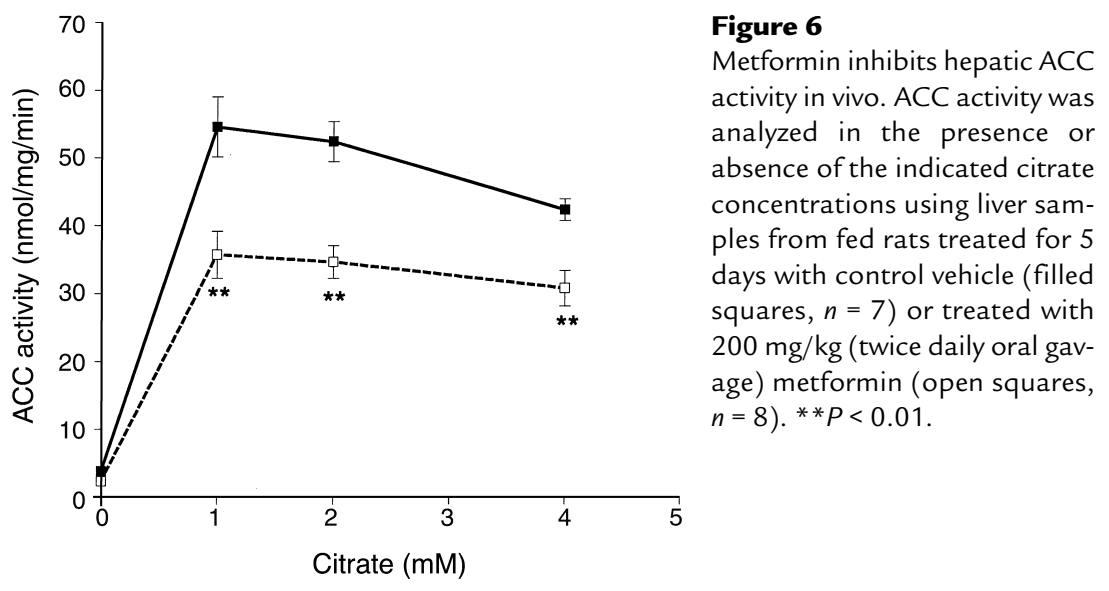

ment with metformin prevented this accumulation. Additional results obtained using hepatic nuclear extracts from re-fed rats after treatment with AICAR $(500 \mathrm{mg} / \mathrm{kg} /$ day $)$ also showed that the presence of SREBP-1 mature-form protein was ablated (data not shown).

Measurement of AMPK activation in liver ex vivo is difficult because brief hypoxia is known to produce marked activation of the enzyme (ref. 37 and data not shown). Thus we used liver tissue derived from metformin-treated rats to determine that ACC activity was decreased significantly at several tested citrate concentrations (Figure $6)$. The greatest ACC activity reduction was at a citrate concentration of $1 \mathrm{mM}$ (from $54.6 \pm 11.8$ to $35.6 \pm 7.7$ $\mathrm{nmol} / \mathrm{mg} / \mathrm{min} ; P<0.01)$. These results are consistent with metformin having produced in vivo AMPK activation and ACC inactivation.

\section{Discussion}

To summarize, results presented here are consistent with a model (Figure 7) in which increased phosphorylation and activation of AMPK by metformin leads to the effects on glucose and lipid metabolism as follows. Phosphorylation and inactivation of ACC, as a result of AMPK activation, serves to inhibit the proximal and rate-limiting step of lipogenesis. Reduced synthesis of the ACC product, malonyl-CoA, is also predicted to relieve inhibition of CPT-1, resulting in increased fatty acid oxidation. These effects are likely to contribute to metformin's in vivo ability to lower triglycerides and VLDL.

Figure 7 metabolism. FA, fatty acid. hepatocyte glucose production by metformin under the test conditions we employed. Additional studies will be required to further elucidate precise mechanism(s) by which metformin-stimulated AMPK activation could result in inhibition of hepatic glucose production.

AMPK activation is implicated as a mechanism for the induction of skeletal muscle glucose uptake; this effect is also additive with insulin (12). Thus, the observed association of increased glucose uptake and AMPK activation in isolated skeletal muscles suggests that metformin's effect to augment muscle insulin action in vivo may be attributed to AMPK as well.

A major mechanism (AMPK activation) by which metformin produces beneficial metabolic effects has been characterized, along with discovery of the mechanism (SREBP-1 suppression), by which AMPK inhibits the expression of lipogenic genes. Attempts to generate novel therapies for metabolic disease via AMPK activation may be worthy of pursuit. ders $(35,36)$ and that increased liver lipid content is implicated in hepatic insulin resistance (38).

Metformin-mediated effects on hepatic glucose production contribute to its glucose-lowering efficacy. We demonstrated (using an AMPK inhibitor, Figure 3) that AMPK activation is required for inhibition of

\section{Acknowledgments}

We thank Shiying Chen for characterizing anti-AMPK Ab's, Marcie Donnelly for technical support related to animal studies, Mark Fraley for compound synthesis, and Georgianna Harris and Denis McGarry for helpful discussions and intellectual support.

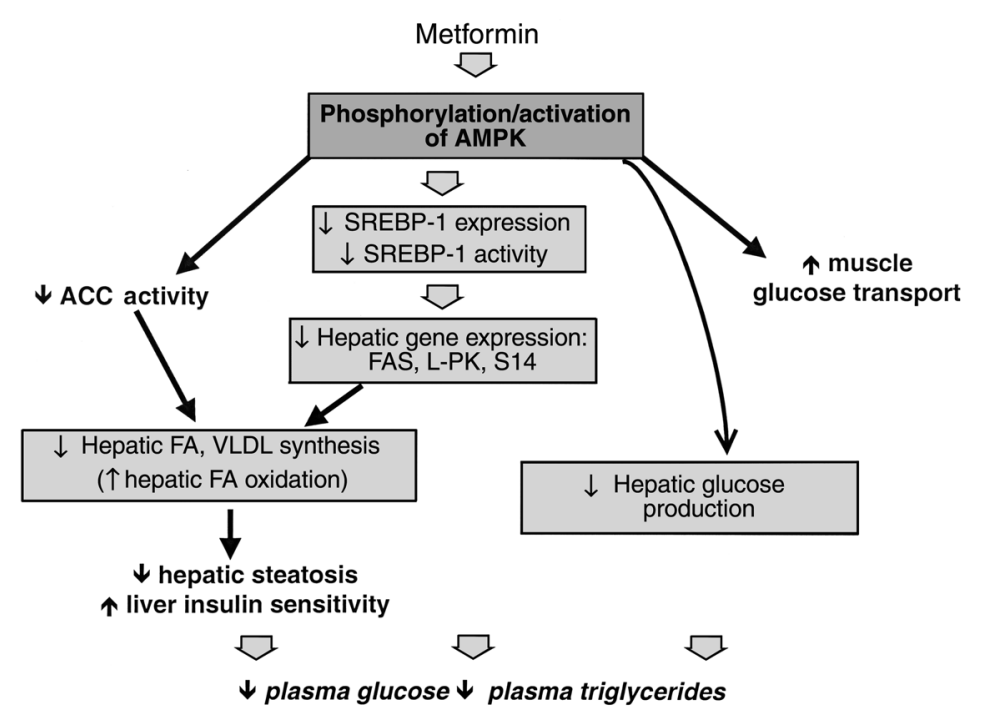

Model for the mechanism by which metformin mediates effects on lipid and glucose 
1. 2000. IMS HEALTH's National Prescription Audit Plus ${ }^{\mathrm{TM}}$. http://www.ims-global.com.

2. Stumvoll, M., Nurjhan, N., Perriello, G., Dailey, G. and Gerich, J.E. 1995. Metabolic effects of metformin in non-insulin-dependent diabetes mellitus. N. Engl. J. Med. 333:550-554.

3. Wiernsperger, N.F., and Bailey, C.J. 1999. The antihyperglycaemic effect of metformin: therapeutic and cellular mechanisms. Drugs. 58:31-39.

4. Wu, M.S., et al. 1990. Effect of metformin on carbohydrate and lipoprotein metabolism in NIDDM patients. Diabetes Care. 13:1-8.

5. Schafer, G. 1983. Biguanides. A review of history, pharmacodynamics and therapy. Diabete Metab. 9:148-163.

6. Hundal, R.S., et al. 2000. Mechanism by which metformin reduces glucose production in type 2 diabetes. Diabetes. 49:2063-2069.

7. Hundal, H.S., Ramlal, T., Reyes, R., Leiter, L.A., and Klip, A. 1992. Cellular mechanism of metformin action involves glucose transporter translocation from an intracellular pool to the plasma membrane in L6 muscle cells. Endocrinology. 131:1165-1173.

8. Galuska, D., Nolte, L.A., Zierath, J.R., and Wallberg-Henriksson, H. 1994. Effect of metformin on insulin-stimulated glucose transport in isolated skeletal muscle obtained from patients with NIDDM. Diabetologia. 37:826-832.

9. Lin, H.Z., et al. 2000. Metformin reverses fatty liver disease in obese, leptin-deficient mice. Nat. Med. 6:998-1003.

10. Hardie, D.G., and Carling, D. 1997. The AMPactivated protein kinase: fuel gauge of the mammalian cell? Eur. J. Biochem. 246:259-273.

11. Winder, W.W., and Hardie, D.G. 1999. AMP-activated protein kinase, a metabolic master switch: possible roles in type 2 diabetes. Am. J. Physiol. 277:E1-E10.

12. Hayashi, T., Hirshman, M.F., Kurth, E.J., Winder, W.W., and Goodyear, L.J. 1998. Evidence for 5' AMP-activated protein kinase mediation of the effect of muscle contraction on glucose transport. Diabetes. 47:1369-1373

13. Merrill, G.F., Kurth, E.J., Hardie, D.G., and Winder, W.W. 1997. AICA riboside increases AMP-activated protein kinase, fatty acid oxidation, and glucose uptake in rat muscle. Am. J. Physiol. 273:E1107-E1112.

14. Goodyear, L.J. 2000. AMP-activated protein kinase: a critical signaling intermediary for exer- cise-stimulated glucose transport? Exerc. Sport Sci. Rev. 28:113-116.

15. Lochhead, P.A., Salt, I.P., Walker, K.S., Hardie, D.G., and Sutherland, C. 2000. 5-aminoimidazole-4-carboxamide riboside mimics the effects of insulin on the expression of the 2 key gluconeogenic genes PEPCK and glucose-6-phosphatase. Diabetes. 49:896-903.

16. Foretz, M., Carling, D., Guichard, C., Ferre, P and Foufelle, F. 1998. AMP-activated protein kinase inhibits the glucose-activated expression of fatty acid synthase gene in rat hepatocytes. $J$. Biol. Chem. 273:14767-14771.

17. Holmes, B.F., Kurth-Kraczek, E.J., and Winder, W.W. 1999. Chronic activation of $5^{\prime}$-AMP-activated protein kinase increases GLUT-4, hexokinase, and glycogen in muscle. J. Appl. Physiol 87:1990-1995.

18. Pollard, J.W., and Walker, J.M., editors. 1992. Basic cell culture protocols. Methods in Molecular Biology series, Volume 75. Humana Press Inc. Totowa, New Jersey, USA. 145-150.

19. Witters, L.A., and Kemp, B.E. 1992. Insulin activation of acetyl-CoA carboxylase accompanied by inhibition of the $5^{\prime}$-AMP-activated protein kinase. J. Biol. Chem. 267:2864-2867.

20. Davies, S.P., Carling, D., and Hardie, D.G. 1989 Tissue distribution of the AMP-activated protein kinase, and lack of activation by cyclic-AMPdependent protein kinase, studied using a specific and sensitive peptide assay. Eur. J. Biochem. 186:123-128.

21. Mannaerts, G.P., Debeer, L.J., Thomas, J., and DeSchepper, P.J. 1979. Mitochondrial and peroxisomal fatty acid oxidation in liver homogenates and isolated hepatocytes from control and clofibrate-treated rats. J. Biol. Chem. 254:4585-4595.

22. Carling, D., Clarke, P.R., Zammit,V.A., and Hardie, D.G. 1989. Purification and characterization of the AMP-activated protein kinase. Copurification of acetyl-CoA carboxylase kinase and 3-hydroxy-3-methylglutaryl-CoA reductase kinase activities. Eur. J. Biochem. 186:129-136.

23. Hayashi, T., et al. 2000. Metabolic stress and altered glucose transport: activation of AMP-activated protein kinase as a unifying coupling mechanism. Diabetes. 49:527-531.

24. Sheng, Z., Otani H., Brown, M.S., and Goldstein, J.L. 1995. Independent regulation of sterol regulatory element-binding proteins 1 and 2 in hamster liver. Proc. Natl. Acad. Sci. USA. 92:935-938.
25. Cusi, K., and DeFronzo, R.A. 1998. Metformin: a review of its metabolic effects. Diabetes Reviews 6:89-131.

26. Wiernsperger, N.F. 1999. Membrane physiology as a basis for the cellular effects of metformin in insulin resistance and diabetes. Diabetes. Metab. 25:110-127.

27. Sum, C.F., et al. 1992. The effect of intravenous metformin on glucose metabolism during hyperglycaemia in type 2 diabetes. Diabet. Med. 9:61-65.

28. Wilcock, C., Wyre, N.D., and Bailey, C.J. 1991. Subcellular distribution of metformin in rat liver. J. Pharm. Pharmacol. 43:442-444

29. Wilcock, C., and Bailey, C.J. 1994. Accumulation of metformin by tissues of the normal and diabetic mouse. Xenobiotica. 24:49-57.

30. Hermann, L.S. 1979. Metformin: a review of its pharmacological properties and therapeutic use. Diabete Metab. 5:233-245.

31. Sterne, J., Duval, D., and Junien, J.L. 1979. Metformin therapy. Research Clinical Forums 1: 13.

32. Owen, M.R., Doran, E., and Halestrap, A.P. 2000 Evidence that metformin exerts its anti-diabetic effects through inhibition of complex 1 of the mitochondrial respiratory chain. Biochem. J. 348:607-614.

33. El-Mir, M.Y., et al. 2000. Dimethylbiguanide inhibits cell respiration via an indirect effect targeted on the respiratory chain complex I. J. Biol. Chem. 275:223-228.

34. McGarry, J.D., and Brown, N.F. 1997. The mitochondrial carnitine palmitoyltransferase system. From concept to molecular analysis. Eur. J. Biochem. 244:1-14.

35. Shimomura, I., et al. 2000. Decreased IRS-2 and increased SREBP-1c lead to mixed insulin resist ance and sensitivity in livers of lipodystrophic and ob/ob mice. Mol. Cell. 6:77-86.

36. Kakuma, T., et al. 2000. Leptin, troglitazone, and the expression of sterol regulatory element binding proteins in liver and pancreatic islets. Proc Natl. Acad. Sci. 97:8536-8541.

37. Davies, S.P., Carling, D., Munday, M.R., and Hardie, D.G. 1992. Diurnal rhythm of phosphorylation of rat liver acetyl-CoA carboxylase by the AMP-activated protein kinase, demonstrated using freeze-clamping. Effects of high fat diets. Eur. J. Biochem. 203:615-623.

38. McGarry, J.D. 1992. What if Minkowski had been ageusic? An alternative angle on diabetes. Science. 258:766-770. 